\title{
Overview and Analysis of UWB signal for Low Power WPAN
}

\author{
Rajesh Thakare \\ Assistant Professor \\ Dept.of Electronics Engg. \\ Priyadarshini College of Engg. Nagpur
}

\author{
Kishore Kulat \\ Professor \\ Dept.of Electronics and Computer \\ Science Engg.VNIT,Nagpur
}

\author{
Santosh Chede \\ Assistant Professor \\ Dept.of Electronics and Telcom. \\ Priyadarshini College of Engg.Nagpur
}

\begin{abstract}
In modern short range wireless applications, RF, Bluetooth and UWB communication schemes are recommended with respect to power consumption, low/high data rates and location capability. Though special bandwidth is not allocated to UWB, coexistence of such signal in the available RF environment is a critical issue and hence analysis of UWB signal with respect to interference is very much required in dedicated WPAN networks. In this paper, effect of UWB interference for existing systems such as GPS, FWA, UMTS, DCS1800 is analyzed. Communication path distance is directly proportional to the UWB signal pulse width. To correctly define the UWB signal this functionality is realized by composing UWB signal and results are simulated using MATLAB.
\end{abstract}

\section{Keywords}

Communication systems, UWB, WPAN

\section{INTRODUCTION}

UWB is a fast emerging technology used for future short range indoor radio communication. This system provides very high bit rates services, low power consumption and accuracy position capability[1].A significant difference between traditional radio transmissions and UWB radio transmissions is that traditional systems transmit information by varying the power level, frequency, and/or phase of a sinusoidal wave. UWB transmissions transmit information by generating narrow pulses of width of nanosecond at specific time instants and occupying large bandwidth thus enabling a pulse-position or time-modulation $[2,9]$. As system occupies very large bandwidth the same bandwidth is also used by other existing communication systems. To have guarantee, existing communication system from UWB emission, the federal communication commission (FCC) restricted the UWB operating band in the $3.1-10.6 \mathrm{GHz}$ frequency range and regulated UWB power emission with frequency-power mask issued for each specific UWB application.[3].After the authorization by FCC,IEEE has incorporated UWB into technical standard for high rate WPANS ,Low rate WPAN and Body Area Network[4].

UWB technology holds great promise for a vast region of new applications that provide significant benefits for public safety, businesses and consumers. The next generation of $\mathrm{PC}$, consumer

electronics and mobile application demand connectivity speed beyond $1 \mathrm{Mbps}$ peak data rate of Bluetooth Technology, which is used by many devices to create WPAN today. But many CE devices cannot support the cost and power required by higher seed networking.UWB provides the throughput required by next generation of converged devices [5].

Under appropriate technical standards, UWB devices operate at the same spectrum already occupied by existing radio services, thereby allowing scarce spectrum resources to be used more efficiently UWB system is classified using one of two different measures of bandwidth as per the FCC. A system can either have an instantaneous bandwidth in excess of $500 \mathrm{MHz}$ or have a fractional bandwidth that exceeds 0.20 (by comparison a narrowband signal typically has a fractional bandwidth which is less than 0.01). Both cutoff frequencies are defined according to the $-10 \mathrm{~dB}$ points of the signal's spectrum. Fractional bandwidth is defined as the signal's bandwidth divided by its center frequency or more precisely as

$$
B w f=2 * \frac{f h-f l}{f h+f l}
$$

Where,

$f h$ - highest frequency

$f l$ - lowest frequency of the signal at the $-10 \mathrm{~dB}$ points [FCC].

These definitions specify that systems with a center frequency greater than $2.5 \mathrm{GHz}$ must have a bandwidth greater than 500 $\mathrm{MHz}$ and a system with a center frequency less than $2.5 \mathrm{GHz}$ must have a fractional bandwidth greater than 0.20 . Figure 1 below provides an illustration comparing the fractional bandwidth of a narrowband signal and a UWB signal, $B W_{N B}$ is the narrowband signal bandwidth, $B W_{U W B}$ is the UWB signal bandwidth, and fc is the signal's center frequency.

\subsection{UWB concept}

UWB transmissions transmit information by generating radio energy at specific time instants and occupying large bandwidth thus enabling a pulse-position or time-modulation. The information can also be modulated on UWB pulses by encoding the polarity of the pulse, the amplitude of the pulse, and/or by using orthogonal pulses. UWB pulses can be sent sporadically at 
relatively low pulse rates to support time/position modulation, but can also be sent at rates up to the inverse of the UWB pulse bandwidth. Pulse shape is important for UWB because it is a baseband technology and spectrum is determined by pulse shape and pulse width. A UWB pulses are generated by Gaussian monopulse, Gaussian second derivative and RZ Manchester.[1,8]. Another valuable aspect of pulse-based UWB is that the pulses are very short in space (less than $60 \mathrm{~cm}$ for a $500 \mathrm{MHz}$ wide pulse, less than $23 \mathrm{~cm}$ for a $1.3 \mathrm{GHz}$ bandwidth pulse), so most signal reflections do not overlap the original pulse, and thus the traditional multipath fading of narrow band signals does not exist. However, there still is multipath propagation and inter-pulse interference for fast pulse systems which can be mitigated by coding techniques.

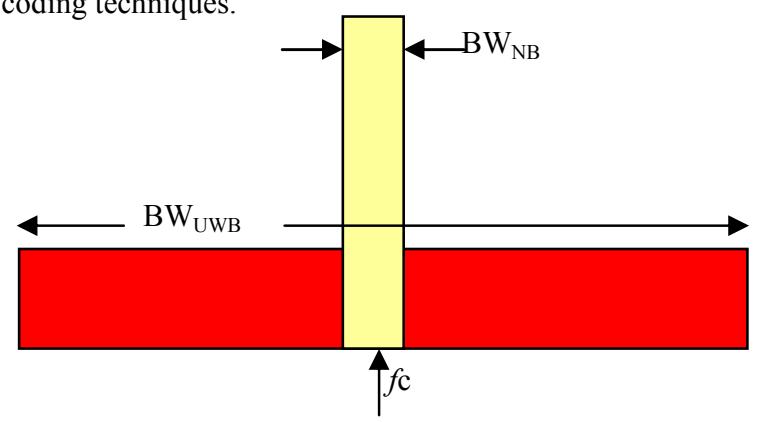

Figure1. Comparison of Fractional bandwidth a narrowband and UWB signal

\subsection{FCC rules for UWB communication}

UWB devices use a low transmission power spectral density in order to, not interfere with existing narrowband communications systems. For this reason the FCC has provided a preliminary conservative spectral mask for all UWB systems. Current FCC Regulations in order to protect existing radio services from UWB interference, the FCC has assigned conservative emission masks between $3.1 \mathrm{GHz}$ and $10.6 \mathrm{GHz}$ for commercial UWB devices. The maximum allowed power spectral density (PSD)for these devices that is, $-41.3 \mathrm{dBm} / \mathrm{MHz}$, or $75 \mathrm{nW} / \mathrm{MHz}$ places them at the same level as unintentional radiators (FCC Part 15 class) such as televisions and computer monitors. For particularly sensitive bands such as the global positioning system (GPS) band (0.96 to $1.61 \mathrm{GHz}$ ), the PSD limit is much lower. As depicted in Fig. 2, such a ruling allows UWB devices to overlay existing narrowband systems while ensuring sufficient, attenuation to limit adjacent channel interference [6,7]. Equivalent isotropically radiated power, i.e., the product of the power supplied to the antenna and the antenna gain in a given direction relative to an isotropic antenna. The EIRP, in terms of $\mathrm{dBm}$, Ultra wideband offers new capabilities for short-range communications, ground- and object penetrating radar, vehicular radar, security systems and measurement applications

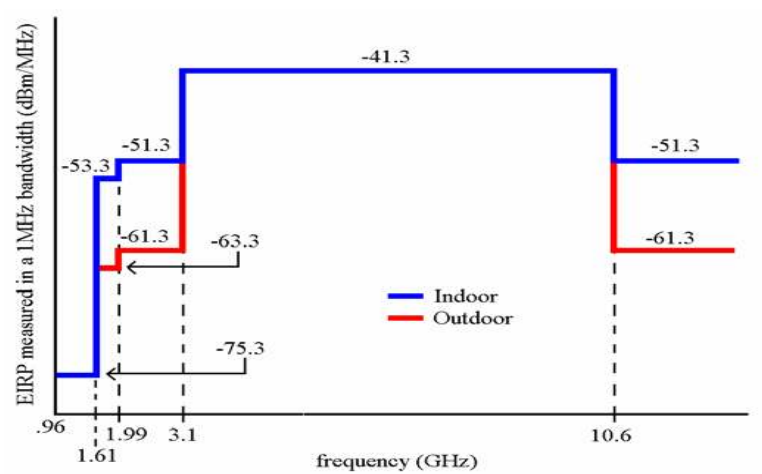

Figure 2. FCC Spectral Mask for Communications and Measurements Applications

\section{ANALYSIS OF UWB INTERFERENCE LIMITS}

UWB based communication consists of transmitter and receiver. In such systems, UWB signal must be transmited without affecting the performance of other systems operating in its coexistence. Implantation of UWB transmitter and receiver do not require expensive and large components, such as modulators, demodulators, and IF stages. This fact can reduce cost, size, weight, and power consumption of UWB systems in comparison with conventional narrowband communication systems. Effect of existence of UWB signal transmission in the presence of other receiver is analyzed.

Let the received power given by

$P_{R X}(d)=P_{T X} G_{T X} G_{R X}\left(\frac{\lambda}{4 \pi d}\right)^{2}$

Power spectral density is

$\mathrm{N}_{0}=\mathrm{K}_{\mathrm{B}} \mathrm{T}_{\mathrm{e}}$

Noise power is

$P n=N o B$

Thermal noise power contained in bandwidth $\mathrm{B}$ is

$N=-144+10 \log B+N f \quad \mathrm{dBW}$

Where

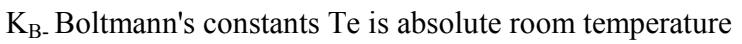

$\mathrm{B}-$ Bandwidth of victim receiver in $\mathrm{MHz}$.

$\mathrm{Nf}$ - noise figure.

The performance of Victim receiver is obtained by finding the signal to noise ratio with and without UWB interference. If we consider the SNRo is signal to noise interference without UWB interference and the SNR is signal to noise interference UWB interference.

$$
\begin{aligned}
& S N R o=\frac{S}{N} \\
& S N R=\frac{S}{I U W B+N}
\end{aligned}
$$


As a result ,the performance of receiver degrades due to UWB interference.This degradation is calculated as follows

$$
d=\frac{I U W B+N}{N}
$$

The interference issue is on the proximity; at the distances that UWB and communication system could interact, there are at least two solutions that could be employed : Detect and Avoid (DAA) and collaborative coexistence. DAA can be used at distances of a few meters of separation between the communication-enabled device and the UWB enabled device. In order to find what amount of maximum UWB interference can be tolerated by communication systems so that the operation of victim receiver is not affected. The maximum allowable UWB interference limits are shown in table 1 .

Table I UWB Allowable Interference Limit

\begin{tabular}{|l|l|}
\hline System & IUWB \\
\hline FWA & $-142 \mathrm{dBW}$ \\
\hline pp & $-142 \mathrm{dBW}$ \\
\hline UMTS & $-134 \mathrm{dBW}$ \\
\hline DCS1800 & $-148 \mathrm{dBW}$ \\
\hline GPS & $-155 \mathrm{dBW}$ \\
\hline
\end{tabular}

\section{EXPERIMENTAL RESULTS}

Simulation Result for Rectangle Pulse of $0.1 \mathrm{~s}$ gives $f l=0 \mathrm{~Hz}$, $f h=6 \mathrm{~Hz}$ and $3 \mathrm{~dB}$ band width is $6 \mathrm{~Hz} \& f l=0 \mathrm{~Hz}, f h=8 \mathrm{~Hz}$ and $10 \mathrm{~dB}$ band width is $8 \mathrm{~Hz}$. This is represented in figure $1 \& 2$ respectively.

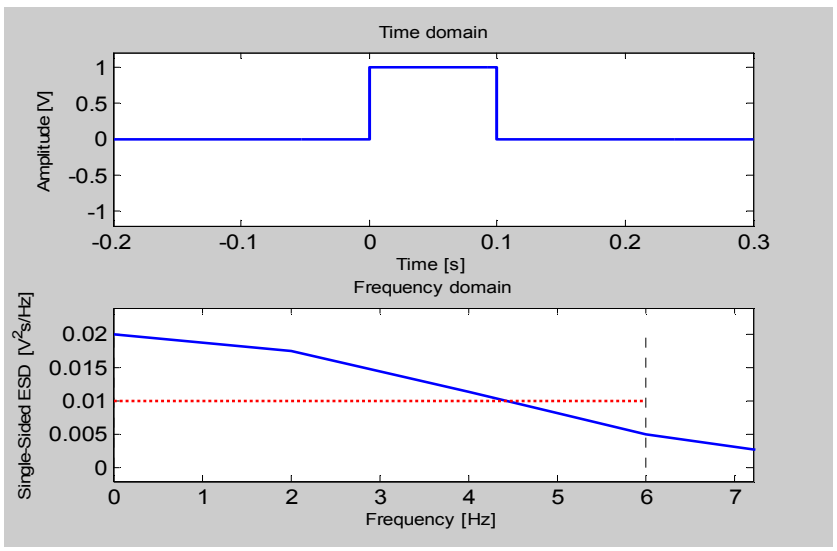

Figure 1. 3dB signal bandwidth for rectangular pulse

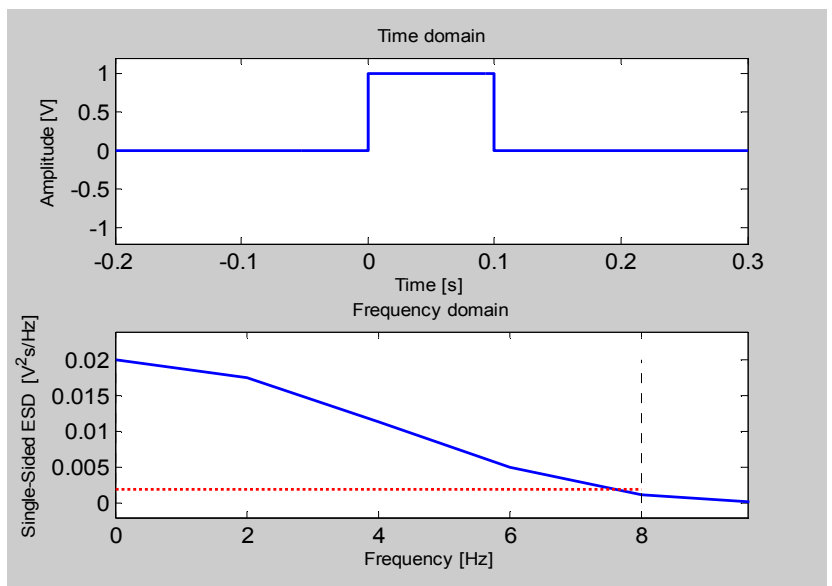

Figure 2. $10 \mathrm{~dB}$ signal bandwidth for rectangular pulse

Simulation Result for Rectangle Pulse of 0.1s is modulated with Sinusoidal of $1 \mathrm{KHz}$ gives $f l=992 \mathrm{~Hz}, f h=1008 \mathrm{~Hz}$ and $10 \mathrm{~dB}$ band width is $16 \mathrm{~Hz}$ shown in figure 3 .

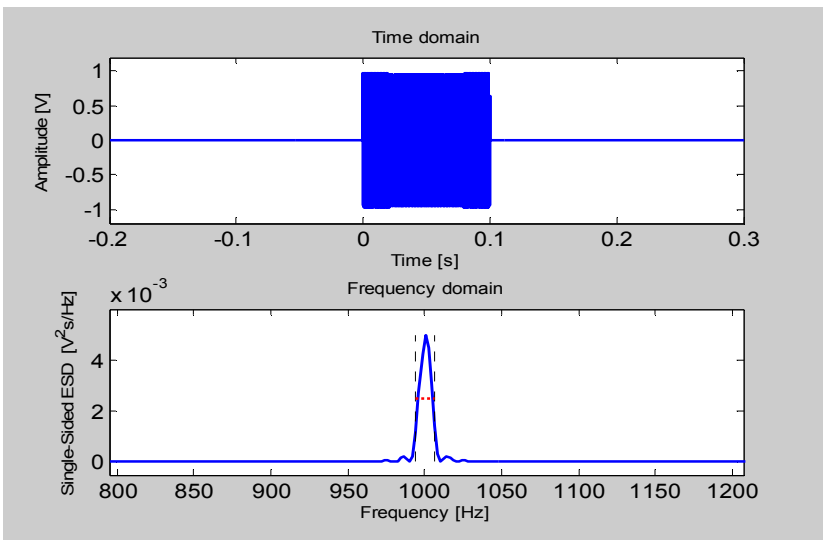

Figure 3. A 10dB Bandwidth Rectangle Pulse modulated with sinusoidal signal

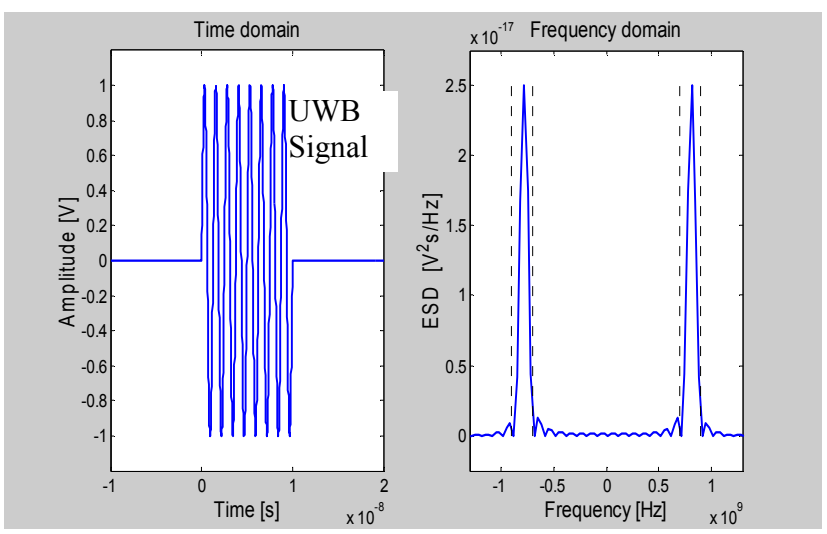

Figure 4. Recognition of UWB Signal

Central Frequency $=0.800000[\mathrm{GHz}]$ 
Bandwidth $=0.200000[\mathrm{GHz}]$

Fractional Bandwidth $=0.250000$
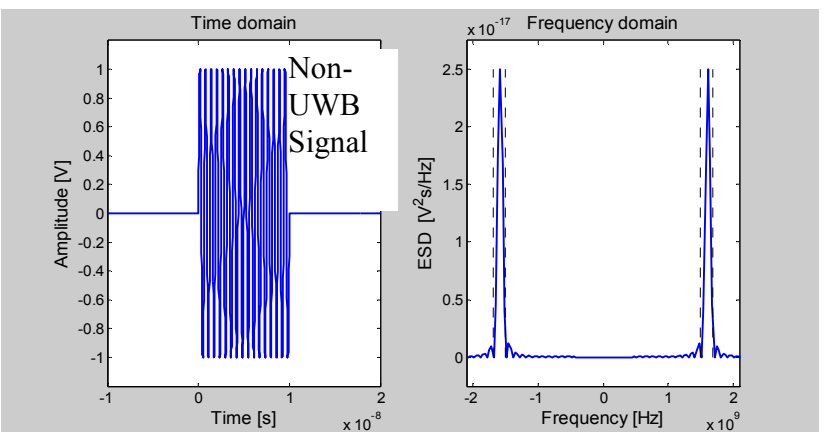

Figure 4. Recognition of Non-UWB Signal

Central Frequency $=1.600000[\mathrm{GHz}]$

Bandwidth $=0.200000[\mathrm{GHz}]$

Fractional Bandwidth $=0.125000$

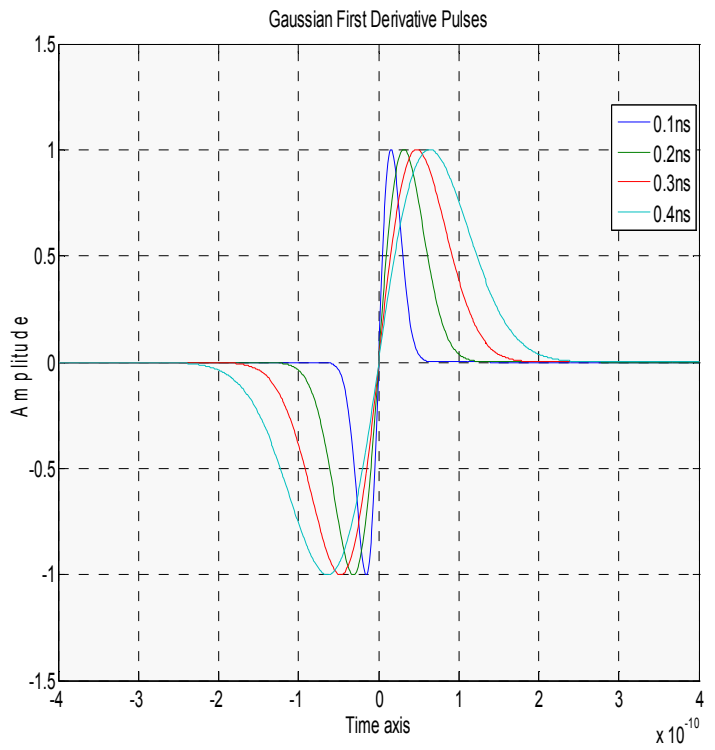

Figure 5. First derivative Gaussian pulse for Various pulse Duration
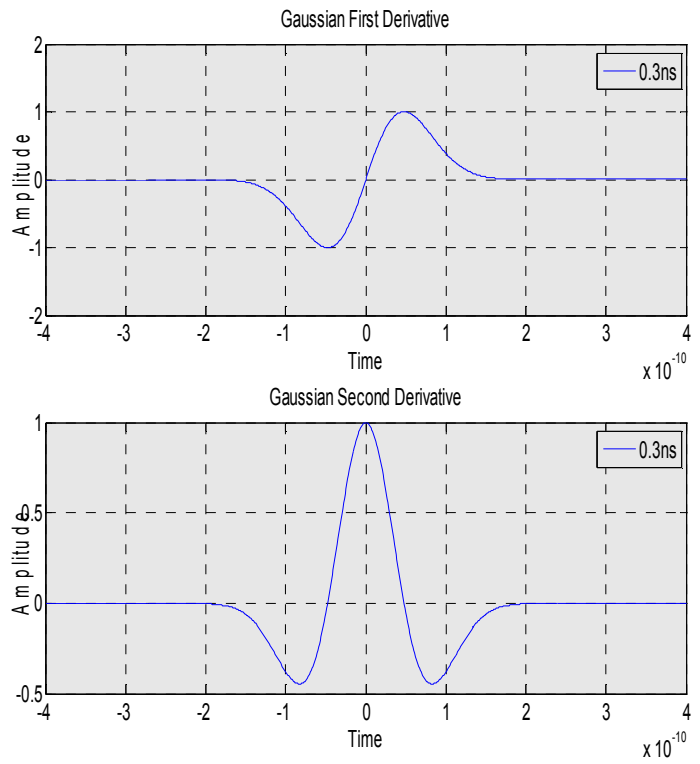

Figure 6. First and second Derivative Gaussian Pulse

Simulation result to classify UWB signal depending on Fractional bandwidth is shown in figure 4 and 5 respectively. As Fractional Bandwidth is greater than 0.2 the given sine pulse is a UWB signal. Fractional bandwidth shown in figure 4, is $0.25 \mathrm{GHz}$, which is greater than 0.2. Hence signal is UWB signal. Similarly Fractional bandwidth shown in figure 5 is $0.125 \mathrm{GHz}$, which is less than 0.2. Hence this is not a UWB signal. Various pulses with reference to Gaussian first order and second order derivatives as shown in figure 5 and 6 can be used as a source signal to test UWB signal status.

\section{CONCLUSION}

From simulation it is clear that Bandwidth occupied by modulated signal is twice the value evaluated in base band for $3 \mathrm{~dB} \& 10 \mathrm{~dB}$. As Fractional Bandwidth is less than 0.2 the given sine pulse is not a UWB signal even though Bandwidth.\& pulse duration is same because Fractional bandwidth is decreased due to the shift of signal spectrum to higher frequency.

\section{REFERENCES}

[1] Romeo Giuliano, Gianlua Guidoni, Franco Mazzenga and Francesco Vatalaro, " On UWB Coexistance with UMTS Terminals, "IEEE Communication Society, 2004,3571-3575.

[2] Abdul Zaman and Nazmul Islam" Modulation Schemes and Pulse Shaping In Ultra - Wideband "IEEE Transactions on Communications, pp. 142-146,2008.

[3] Federal Communications Commission, "Revision of Part 15 of the commission's rules regarding ultra-wideband transmission system," First Report and Order, ET Docket 98-153, FCC 02-48, February 14,2002

[4] Huilin Xu and Liuqing Yang, "Ultra-Wideband Technology: Yesterday, Today, and Tomorrow," IEEE Transactions on Communications, 2008, 715-718 
[5] White paper on; "Ultra-Wideband Technology" in, Intel in Communication.

[6] Gary Breed, "A summary of FCC rules for Ultra wideband Communications".

[7] Khodayar Sarafaraz, Seyed Ali, Ghavami "Evaluating the interference effect on DS-UWB System on Wi-Max System," IEEE Transactions on Communications, 2006.
[8] Brent Parr, Byunglok Cho, Kenneth Wallace et al. "A Novel Ultra-Wideband Pulse Design Algorithm," IEEE Communications Letters,vol.7, no.5, 2003, 219-221

[9] Zou Weixia, Zhang Chunqing, Zhou Zheng. "Algorithm to design UWB pulse based on the peak frequency," Journal on Communications, vol.26,no.9, September 2005,74-78

[10] Igor Dotlic, Ryuji Kohno. "Design of the family of orthogonal and spectrally efficient UWB waveforms," IEEE Journal of Selected Topics in Signal Processing, vol.1, no. 1, June 2007,21-30. 